\title{
Integrating Qualitative Comparative Analysis and Support Vector Machine Methods to Reduce Passengers' Resistance to Biometric E-Gates for Sustainable Airport Operations
}

\author{
Cheong Kim ${ }^{1,2} \mathbb{D}$, Francis Joseph Costello ${ }^{1}(\mathbb{D})$ and Kun Chang Lee ${ }^{1,3,4, *}$ \\ 1 SKK Business School, Sungkyunkwan University, Seoul 03063, Korea; saga@g.skku.edu (C.K.); \\ f.costello@g.skku.edu (F.J.C.) \\ 2 Korea Airports Corporation (KAC), Seoul 07505, Korea \\ 3 Samsung Advanced Institute for Health Sciences \& Technology (SAIHST), Sungkyunkwan University, \\ Seoul 03063, Korea \\ 4 Creativity Science Research Institute (CSRI), Sungkyunkwan University, Seoul 03063, Korea \\ * Correspondence: leekc@skku.edu
}

Received: 16 August 2019; Accepted: 21 September 2019; Published: 27 September 2019

check for updates

\begin{abstract}
For the sake of maintaining sustainable airport operations, biometric e-gates security systems started receiving significant attention from managers of airports around the world. Therefore, how to reduce flight passengers' perceived resistance to the biometric e-gates security system became much more important than ever. In this sense, the purpose of this study is to analyze the factors which contribute to passenger's resistance to adopt biometric e-gate technology within the airport security setting. Our focus lies on exploring the effects that perceived risks and benefits as well as user characteristics and propagation mechanisms had on causing such resistance. With survey data from 339 airport users, a support vector machine (SVM) model was implemented to provide a tool for classifying resistance causes correctly, and csQCA (crisp set Qualitative Comparative Analysis) was implemented in order to understand the complex underlying causes. The results showed that the presence of perceived risks and the absence of perceived benefits were the main contributing factors, with propagation mechanisms also showing a significant effect on weak and strong resistance. This study is distinct in that it has attempted to explore innovation adoption through the lens of resistance and in doing so has uncovered important complex causation conditions that need to be considered before service quality can be enhanced within airports. This study's implications should therefore help steer airport managers in the right direction towards maintaining service quality while implementing sustainable new technologies within their current airport security ecosystem.
\end{abstract}

Keywords: sustainable airport operation; biometric e-gate security; machine learning; support vector machine (SVM); ensemble classifiers

\section{Introduction}

Global leading airports, such as Incheon International Airport (IIA), are looking to provide improved passenger services in order to maintain the overall airport experience. This has been cited as one of the key factors for developing sustainable airport operations and their own competitiveness [1]. Therefore, passenger service quality within airports are evaluated every year. For example, the Airport Service Quality (ASQ) of Airport Council International (ACI) help to ensure that airports provide appropriate passenger services that are sustainable in the long term. One important component of the passenger service quality measurement is the waiting time. However, airports have started to suffer from various issues recently, including prolonged waiting times to board, and this has subsequently 
decreased the passenger service quality. These are attributable to extreme congestion in passenger terminals. In fact, according to the International Civil Aviation Organization (ICAO), the number of global air-passengers rose by $78 \%$ to 36.96 billion in 2016, up from 20.72 billion in 2006. This is equivalent to an annual growth rate of $7.8 \%$, leading to concerns over the sustainability of the state of current airport operations in the perspective of passenger service quality. Thus, recent attempts to mitigate these problems are an ongoing research problem in sustainable airport operation $[2,3]$. In order to sustain this continuous growth in air passengers, many international airports globally are expanding their facilities, or even building new airports to increase their operation capacity. Other airports have looked to introduce self-service systems, as well as automated devices, such as Self Check-in, Self-Bag-Drop, or Biometric Identification [4]. According to the International Air Transport Association (IATA), the implementation of self-service during the boarding process will increase to $80 \%$ by 2020 [5].

Although automated devices are increasing in power and number, it is not enough. Rising congestion and queuing at airports is a much more complex problem to deal with. Up till now much research has gone into optimization methods in helping to reduce the burden of numbers at airports [3]. One such area that needs optimization, but raises great concern, is the ways in which to sustain the levels of security. This has become something of a top priority for most airports as well as governments. While optimizing security is necessary, there are a few fundamentals of a security network for sustainable airport operations that need to be considered: (1) it needs to be less costly, (2) include better operating procedures, (3) be able to reduce passenger's waiting time, and (4) improve the overall experience for the passengers [3].

In the case of Korea (where this study was based) biometric technology has become a considered sustainable alternative to the common human agents for identification of passengers at pre-security areas in airports. The aim is to reduce congestion by installing biometric e-gates in parallel at these pre-security areas. The need for this technology has been identified due to a 2017 Air Traffic Service Report (Ministry of Land, Infrastructure and Transport, 2017), where they identified that the number of air passengers in Korea had grown rapidly, reaching 109.36 million in 2017, surpassing the 100 million mark for two years in a row. Alongside this, the Korean Airports Corporation (KAC) found in an online study that a safe airport, including security measures, was ranked number one amongst internal (19.6\%) and external (17.4\%) stakeholders, based on sentiment towards social value prioritization [6]. Due to these findings, in January 2018 KAC identified the domestic lines of Gimpo Airport and Jeju Airport as routes for potentially implementing biometric technology. According to a survey of 224 airport users conducted by KAC in October 2017, 78\% of the respondents expected that the biometric boarding system would reduce waiting time and only $8 \%$ of them said that the biometric boarding system would provide no value to the passengers.

With this indication from the public, the biometric e-gate boarding service was introduced by KAC for the Gimpo and Jeju route to enhance passenger service quality. However, contrary to the expectations of high numbers of passengers choosing to use such a service, data released by KAC showed only a total of 12,475 passengers (daily average of 870 passengers) had used the biometric e-gates on this domestic route during January 29 February 13. If we consider that an average of 67,633 passengers used this domestic line on a daily basis in 2017 , it means that only $1.28 \%$ of the passengers used the biometric e-gates. With this realization it became apparent that biometric e-gate security might not be the sustainable system for the future that was much anticipated.

Our research aim, therefore, lies in exploring the reasons why this technology was not yet adopted enthusiastically by air passengers despite the airports' best efforts in providing this beneficial service that will help to sustain low queue times at security areas within airports.

To fulfill this research aim, we adopt two methods: csQCA (crisp set Qualitative Comparative Analysis) and Support Vector Machine (SVM) classification. By implementing csQCA we wanted to explore the underlying complex conditions which support the resistance of biometric e-gate technology. csQCA analysis greatly enables the identification of cases that can be further analyzed for causal process observations. With csQCA not all conditions have to imply a significant meaning, but rather that a 
researcher can select a few cases based on relevant configurations and prior theoretical knowledge [7]. Through csQCA we were able to find relevant conditions that help to support our prior hypothesis and, therefore, can help to provide unique implications, which can help to guide airport managers in implementing similar biometric e-gate technologies.

The SVM methodology, which belongs to intellectual machine-learning algorithms, has been actively used within the field of sustainability research [8-12]. Among the machine-learning algorithms, such as linear discriminate analysis, decision trees, logistic regression, naïve Bayes, artificial neural networks and k-nearest neighbor, SVM is a tried and tested algorithm that has gained much trust amongst academics $[13,14]$. Compared to other machine-learning algorithms, SVM stands out to show greater performance [15-20], by specifically changing kernel function techniques, such as the polynomial kernel, radial basis function (RBF) kernel and Pearson VII universal function (PUF) kernel. Despite this, only a few researches have shed light on these kernel functions used alongside SVM [13]. On top of kernel functions, ensemble classifiers have shown generally better results compared with single classifiers [21]. Hence, it would be expected that SVM classifier ensembles, such as SVM with bagging (BA), random subspace (RSS) and adaptive boosting (ADA), might reveal greater performance than a single SVM classifier in prediction. Therefore, this paper looks to identify the reasons why passengers resist to adopt the biometric e-gate security by exploring the performance of SVM models in combinations of various types of kernel functions and ensemble classifiers as well.

The rest of this research is arranged as follows. In the second section we explore the reasons why people may resist the adoption of technology, specifically biometric e-gate technology as the technology of choice within this paper. Next we will present the first part of our study, the QCA methodology. In particular, an analysis of the csQCA will be made. Additionally, an analysis of SVMs, kernel functions and ensemble techniques will be made before we present the dataset used and assessment metrics applied within both methodologies. Results will then be presented from our empirical testing with both research methods, before a full discussion, conclusions and implications of this research will be made.

\section{Theoretical Background}

\subsection{Biometrics for Passenger Service Quality}

After the 9.11 crisis in 2001, safety and security at airports has become extremely serious and is one of the key tools identified for airports operations [6]. In response to it, most airports have enhanced the level of security, for instance, the US government legislated an extra decree, the Aviation and Transportation Security Act (ATSA), that commanded several momentous changes in aviation security policy [22]. Although this new regulation of screening processes at airports has greatly increased aviation security on the one hand, on the other hand, it also adds to the waiting time of passengers. This endeavor has produced a negative effect on passenger service quality [22]. According to an air passenger survey of 17 countries by SITA in $2015,76 \%$ of passengers claimed that aviation security screening procedures at airports brought forward the most negative reactions in passenger services at the airports [23].

Key stakeholders of the aviation industry, such as airports, airlines and governments, are all aware of this negative response from passengers to the new security policy at airports. Security should not be compromised, however, as it is a very significant matter for the sustainability of the industry. Hence, it is not easy to make compromises for enhancing passenger service quality. Biometric e-gates could be implemented to automate airport security, providing a solution to the paradoxical desire for both improved security and a more productive procedure for passenger service quality to derive sustainable airport operations [23]. Implementing a single-token method can replace the current system and passengers could finish the entire boarding process through the use of their biometric features.

With biometric e-gates, identities of passengers would be automatically identified and examined using their distinct physical biometric features [24]. Because this biometric information is immediately 
received from the passengers, airports could confirm that the passenger is really the same person who is attempting to get approval to enter airside. This would help to reduce identity deception and improve security $[23,24]$. In addition, biometrics at airports could support upgraded passenger services with quicker processing speeds and greater accuracy compared to human agents [25]. Thus, biometric e-gates has become an appreciable substitution of human agents for passenger identification at security entrances, with global airports now aggressively adopting or is in the process of adopting biometric services in parallel at security areas to improve passenger service quality [4].

\subsection{Underlying Theory: Resistance to Innovation}

In an attempt to answer the question of why resistance to biometric e-gate technology was present, we next explore the theory which guided our exploration into this phenomena (refer to Table 3 for a full breakdown of each construct).

As seen in KAC's implementation of biometric e-gate technology, high expectations were not matched. A lack of active use by passengers in the airport showed a resistance to the use of this innovative technology [1]. This indicated that it is not easy to adopt to an innovation despite numerous benefits, such as the elimination of bringing an ID card and reduced waiting time [26]. Moreover, it becomes very difficult to adopt an innovation if the actual user does not accept, or sometimes denies the "benefits" suggested by the providers of a new system. If the users also think that there are serious risk factors in adopting it, there can be a possibility that they even show "resistance" against innovation. In some cases, the majority of the users who do not adopt an innovation might be making a more rational judgment than the few users whose objective is just to adopt innovation [27].

Currently there exists various types of perceived risk which can influence a consumer. This can then lead to resistance from a consumer in adopting a new innovation. Currently, research on resistance has identified the following factors as contributing factors: temporal risk, social risk, physical risk and functional risk [28-34]. Firstly, temporal risk is the concern about an unnecessary waste of one's time that might occur from adopting the new innovation [33]. Social risk is the potential concern that adoption of a new innovative product or service might create a negative impression to others [34]. Physical risks are ones that reflect upon the threat of the new innovative product or service potentially causing bodily harm, inflicting injuries [33]. Functional risks are concerns about whether the functions of the new innovative product or service will actually work properly [33].

Notably, research up till now has focused on the "Diffusion of Innovation" theory originally proposed by Rogers [26]. This theory has been used in various fields when conducting research about innovation. Examples have been seen whereby its use was utilized as a theoretical background when examining the factors that influence the adoption of innovation in education [35] or policies [36]. In the "Diffusion of Innovation" model, it proposes that perceived benefits, such as relative advantage, compatibility and trialability have an important influence on the adoption of innovation [26,35]. In other words, relative advantage is about how much benefit the innovative product or service can provide to an end user compared to the existing products or services on offer in the current marketplace [26]. Compatibility refers to whether the innovation fits the values of the society in which the consumer belongs to. Furthermore, it asks whether it fits the personal experience of the consumer well [26]. Trialability is the concept that says consumers are getting more used to the innovation, or easing anxiety, through pilot use of the product or service [26].

Next, Rogers [37] defined innovation tendency as "the degree of how fast a certain consumer adopts an innovation." Also, Ram [38] presented the consumers' tendency to seek "new things" as one of the main driving factors that can have an effect upon innovation resistance. Additionally, openness to innovation, which refers to the consumers' innovation tendency [39], and an independent decision-making tendency [40] can be seen as a user characteristic that has a potential effect on resistance to innovation.

Moreover, previous studies have highlighted that publicity gained through mass media is a very important way of spreading the acceptance of an innovation throughout a society [26]. The various 
media platforms include online and offline channels which are effective in easing any resistance by consumers through creating a type of intimacy with the innovative product or service [41]. With mass media, negative images of a product or service can be mitigated through the provision of information using mass media outlets [42,43]. In coherence with this notion, this study predicts that the provision of information through online and offline promotions can help to reduce the level of resistance towards a new innovation [32].

However, up till now, previous studies based on the adoption of innovation have not addressed the "resistance" in a variety of forms. In other words, it is highly necessary to conduct research on this factor in order to identify which features influence the adoption of innovation by classifying the types of resistance in the identified forms [32]. As this innovative biometric system is in its early stage of adoption [44], some airports have done more research on the regulations, functions and implementations of biometric e-gates. This paper posits that rather only focusing on these areas, researchers and managers should also concentrate on how to effectively introduce innovations such as biometric e-gate technology to passengers. For example, passengers could simply postpone accepting the biometric e-gate service until it is standardized. On the other hand, passengers could abortively disagree with adopting the service, or even have an effect on other passengers not accepting it. Since there are various types of passengers at airports, it is required to consider different levels of resistance to innovation, such as (1) Weak Resistance and (2) Strong Resistance, in order to attempt to improve the passenger service quality of airports [45].

This research therefore proposes the use of a prediction system that would detect factors which would decrease each different level of resistance of innovation as seen in the biometric e-gate technology to discover the gateway to the solution of passenger service quality enhancement for sustainable airport operations. We have used an SVM in this paper to provide a straightforward and practical solution to identify and help to mitigate resistance of passengers to biometric e-gate services at airports. If this can be successfully achieved, identification of factors that will help to sustain airport operations could be acquired promoting new innovation for passenger service quality improvement [6].

\subsection{Crisp Set Qualitative Comparative Analysis (csQCA)}

Before any manager can implement a successful biometric e-gate security system, they must understand the complex thought processes of air passengers. In our search for a suitable method of analysis, especially one that can capture the underlying complex notions at play, the literature led us to the use of csQCA [46]. csQCA is one part of the overarching QCA social research approach and is best summarized as the original modelling system based on two truth values: True or False [47]. This research approach is based upon set theory and Boolean algebra [32] and allows for "conjunctural causation" across observed cases [48]. Put another way, when implementing QCA research, different constellations of the same factors $(\mathrm{X})$, e.g., theoretical constructs, can lead to the same result on the dependent variable (Y). Specifically, QCA allows for the development of causality while leaving room for complexity in a model, also known as "multiple conjunctural causation" [48]. Thus, different causal "paths" may lead to the same outcome as each path can be just as relevant in its own distinct way compared with other found "paths" [47,48]. Therefore, with the implementation of QCA, a researcher can look for new ideas, propositions or conjectures formulated within the researchers' theory, rather than testing theory already entrenched within prior literature [7].

In this paper, we implement the use of csQCA for three main reasons: (1) The original data was based upon a Likert scale response survey which had a scale from 1-5. Duşa [47] argues binary crisp sets (csQCA) are the most general type of calibrated data in which any kind of raw data can be transformed. Furthermore, due to the bipolar nature of a Likert response scale, the use of fuzzy sets has been questioned due to logical flaws. In an example, this paper's dependent variable resistance is constructed on a Likert response scale from a positive end ( $1=$ low resistance) to a negative end ( 5 = strong resistance). Transforming this bipolar scale into a unidimensional set introduces conceptual flaws. This is because there is no logical reason for which the end "low resistance" can be calibrated 
as a numerical zero point in the set of "resistance". An air passenger who has very low resistance is certainly outside the set of a passenger with high resistance, but the same can be argued about the mid-point $(3=$ neutral to resistance). In csQCA set theory, low resistance and high resistance can be treated as two separate sets, rather than two ends of the same set, like seen in a fuzzy set fsQCA. Thus, implementing csQCA reflects the true nature of responses given in our survey. (2) The number of samples in our dataset was considered within the small- to intermediate- $\mathrm{N}$ size. Previous research has shown that QCA performs most effectively when data samples are within this range and explanatory conditions stay between two and ten [7,46]. Lastly, (3), within this paper we attempted to study the adoption of innovation through "resistance" in two forms-postponement and rejection [32]. As this approach to studying adoption to innovation flips the conceptual lens, we argue that QCA is best placed to capture new ideas or conjectures [7] that previous research has failed to capture while studying adoption through more traditional research methods [26].

The analytical process of QCA is split into four main steps. Once all of these steps are complete, complex solutions for the problem are presented in the form of the model(s) sufficiency of fit $[46,47]$. First, a decision must be made on what cases to include within a model [49]. Second, a data table must be converted into a "truth table" that gives all the possible combinations of the conditions found. A truth table with $\mathrm{K}$ causal conditions leads to $2 \mathrm{~K}$ rows of possible causal combinations [46]. Rows without cases that are empirically significant are logical reminders and those cases where the outcome is both present and absent are considered contradictory configurations. Boolean algebra is then applied in order to present the most parsimonious solution. The parsimonious solution is the smallest common denominator of a causal condition that leads to the same outcome. Lastly, the results can be explored for all the factors that are necessary and sufficient for the outcome: resistance [46,47,49].

The QCA package within the R software environment [47] was used to perform all the tests. First, we had to organize the constructs for testing into two separate files. Our reasoning for this was due to fact that our dependent variable, resistance, was split into two separate constructs and thus gave us "Weak Resistance" (WR) and "Strong Resistance" (SR). These dependent variables were then tested through four identified independent variables, "Perceived Risk" (PR), "Perceived Benefits" (PB), "User Characteristics" (UC), and "Propagation Mechanism" (PM).

The first challenge is how to calibrate data. The data collected within this study is based on a 5-point Likert scale survey. This means the calibration of categorical data is needed, for this reason we choose to use a crisp set calibration $(0,1)$ based on recommendations by [47]. Next, another serious consideration is with the skewed nature of Likert scale responses. As no prior scale based on our constructs came to our knowledge, we failed to find a theoretical solution to calibration. In the absence of ground theoretical solutions for calibration, statistical clustering is a solution. This mathematically looks to find naturally occurring clusters within a given dataset and can be used as a threshold for labelling the data into crisp sets [47].

Next, necessity and sufficiency tests were made. However, first a theoretical definition is needed. Necessity looks to assess whether cases in each outcome have the same causal conditions. In other words, the condition $X$ is necessary when the outcome $Y$ is present alongside the presence of condition $X$. Given this, Y cannot be achieved without the condition X [49,50]. With sufficiency, the opposite logic applies. A condition is considered sufficient if, whenever it is present across each case, the outcome is also present. With sufficiency there should not be any single case whereby it shows the condition but not the outcome. Put differently, if $\mathrm{X}$ is sufficient for $\mathrm{Y}$, then $\mathrm{X}$ cannot exist without the presence of $Y[46]$.

Within QCA there are two main parameters assessing the model's fit: consistency and coverage. Necessity coverage measures the extent to which the elements of an outcome set are also elements of the condition set. Sufficiency consistency measures how much a case shares a given condition or combination of conditions based on a subset of the outcome in question. Sufficiency coverage measures the degree to which a subset relation exists between the outcome and conditions. Likewise, necessity consistency measures the amount that a subset relation exists between the outcome of a found condition (see Ragin [46] and Schneider and Wagemann [49] for a more detailed explanation). 


\subsection{Support Vector Machine (SVM) and Kernels}

SVM was introduced by Vapnik (1998) and has performed greatly in pattern recognition problems [51]. In addition, SVM provides enhanced classification compared to other machine-learning methods [13]. SVM builds a hyperplane for classification that distinguishes between positive $(+1)$ and negative $(-1)$ sets with the greatest margin. [51]. For example, $\left(x_{1}, y_{1}\right),(x 2, y 2) \ldots\left(x_{m}, y_{m}\right)$, where $\mathrm{x}_{\mathrm{i}} \in \mathrm{R}^{\mathrm{d}}$ signifies vectors in a d-dimensional hyperplane and $\mathrm{y}_{\mathrm{i}} \in[-1,+1]$ is a label of the class. Next, SVM indications are generated by transforming the input vectors into a new higher dimensional analogue for the plane in dimensions marked as $\Phi: \mathrm{R}^{\mathrm{d}} \rightarrow \mathrm{H}^{\mathrm{f}}$ where $\mathrm{d}<\mathrm{f}$ [13]. The optimal hyperplane is then formed using a kernel $\mathrm{K}\left(\mathrm{x}_{\mathrm{i}}, \mathrm{x}_{\mathrm{j}}\right)$, which is the product of input vectors $\mathrm{x}_{\mathrm{i}}$ and $\mathrm{x}_{\mathrm{j}}$ and where $\mathrm{K}\left(\mathrm{x}_{\mathrm{i}}, \mathrm{x}_{\mathrm{j}}\right)$ $=\Phi\left(\mathrm{x}_{\mathrm{i}}\right) \cdot \Phi\left(\mathrm{x}_{\mathrm{j}}\right)[13,52]$.

In the modified space, the training instance closest to the hyperplane is called the support vector. The number of such support vectors is typically small relative to the extent of the training set and decides the blanks and crystalline surface of the hyperplane. Among SVM kernels, the Gaussian radial basis function (RBF), polynomial and Pearson VII universal function (PUF) kernels show immense performance, which are $K_{\text {poly }}\left(x_{i}, x_{j}\right)=\left(x_{i} \cdot x_{j}+1\right)^{p}\left(p\right.$ is the degree of polynomial), $K_{G a u s s i a n}\left(x_{i}, x j\right)=$ $\mathrm{e}^{\frac{\left\|x_{i}-x_{j}\right\|^{2}}{2 \sigma}}\left(\sigma\right.$ is Gaussian sigma), and $\mathrm{K}_{\text {Pearson }}\left(\mathrm{x}_{\mathrm{i}}, \mathrm{xj}\right)=1 /\left[1+\left(2 \sqrt{\left|x_{i}-x_{j}\right|^{2} \sqrt{2^{(1 / \omega)}}}-1 / \sigma\right) 2^{\omega}\right]$ ( $\sigma$ and $\omega$ are the half-width and the tailing factor of the peak). Selection of the adequate kernel for SVM highly depends on the dataset and domain of researches. Nonetheless, polynomial and radial basis function (RBF) kernels are frequently employed for miscellaneous purposes [53-57], while the Pearson VII universal function (PUF) kernel is vigorous and has stronger mapping power than the other kernels [58].

\subsection{Synthesis of Recent SVM Literature}

Recent studies on SVM have several limitations. First, most studies have used the RBF kernel function exclusively, despite the possibility of other kernel functions. Second, most related works have focused on accuracy as the main performance metric. However, it is considerable that datasets of prediction are in multiple classes of an imbalanced nature. Therefore, it is inappropriate to rely on just accuracy for deciding the performance of a model. Table 1 below shows recent studies on predictions using SVM in terms of the kernels and evaluation methods appointed.

Table 1. Recent studies on prediction using a support vector machine (SVM).

\begin{tabular}{ccc}
\hline Studies & Kernels & Evaluation \\
\hline Zu et al. [8] & Optimal & Precision \\
Alimi et al. [9] & Linear, Poly, RBF & Precision, Recall, F-Score \\
Xue et al. [10] & RBF & Accuracy \\
Olivares-Mercado et al. [12] & RBF & Precision, Recall, Accuracy, F-Score \\
Joshi et al. [59] & RBF & Accuracy \\
Ahmad et al. [16] & RBF & Accuracy \\
Aruna et al. [60] & RBF & Accuracy \\
Abdelaal et al. [15] & RBF & AUC \\
You and Rumbe [20] & Poly, RBF, Sigmoid & Accuracy \\
Huang et al. [17] & RBF & Accuracy \\
\hline
\end{tabular}

Performance measurements on prediction models using SVM should also include the F-Score and AUC. When used alongside accuracy, especially for complex prediction models including multiclass and imbalanced classification tasks. Accuracy is used to show a model's true performance in a classification task. When this is considered, Accuracy can be easily understood and can quickly represent the performance of each individual outcome. Accuracy can be measured by calculating the confusion matrix in the following way: 


$$
\frac{T P+T N}{T P+F P+T N+F N} \text {. }
$$

Solely relying on accuracy is not enough as overfitting can be an issue. For this reason, the F-score metric can be used with accuracy. The F-score gives the results of the harmonic mean of precision and recall. Precision is the result of all the accurate positives and recall being the number of accurate positive results divided by the relevant samples. Its best value is seen at one and can be measured in the following way:

$$
2 \times \frac{\text { Precision } \times \text { Recall }}{\text { Precision }+ \text { Recall }} .
$$

In addition, Area Under the Curve (AUC) can be used with accuracy and the F-score in order to improve confidence of the evaluation model. The AUC uses two distinct metrics and plots them in order to find out how well a model can classify true results. The curve plots two parameters; recall or true positive rate and the false positive rate. These could be represented in the following way:

$$
T P R=\frac{T P}{T P+F N}, \quad F P R=\frac{F P}{F P+T N} .
$$

The AUC curve displays TPR against FPR at different classification thresholds and aggregates a performance measurement for all possible classification thresholds. Another way to view this metric is through the model's interpretation of the probability that the model ranks a random positive instance more highly than a random negative instance.

In this study, accuracy, the F-score and AUC will be used as evaluation metrics to extract the best performance on predicting "resistance" against innovation based on biometric e-gates.

\subsection{Ensemble Classifiers}

Ensemble classifiers are coalescence of various classifiers and can provide enhanced performance when implementing them against single classifiers [21]. Ensemble classifiers are based on the modular processing of the brain [13]. In addition, each function can be segregated into subdivisions without mutual intervention [61]. Ensemble classifier use the idea of the divide-and-conquer algorithm, which is based on the multi boughed recursive function in computer science. This algorithm recursively breaks down intractable problems into more recognizable problems to use more adequate techniques for each sub-problem. There are three common techniques of ensemble classifiers; Bagging (BA), Random Subspace (RSS) and Adaptive Boosting (ADA) [62].

Bagging, also known as bootstrap aggregating, produces $n$ new datasets from the original dataset for training to build $n$ individual models by random sampling with replacement. Then, $n$ models are aggregated to generate an output average by using a blending approach, for example, majority voting [63] for improving the stability and accuracy of the model.

Random Subspace (RSS), also called attribute bagging [64], reinforces performance of classifications by reducing the correlation between prediction values of datasets with randomly chosen samples [65]. RS constructs a classifier using a decision tree that preserves veracity on training sets. This classifier has several trees that are built by quasi-random selection for training with replacement to derive posterior probabilities.

The curse of dimensionality occasionally occurs to hyperplane machine-learning cases [66]. AdaBoost (ADA), also known as adaptive boosting, only chooses features with high predictive probability, reducing the dimensionality as well as improving the running time. At first, each classifier has the same weight, then, the weight of each trained subset will be changed according to the significance [13]. After replaying this process, the model will make the final result according to the weight of the classifiers [67]. 


\section{Methodology}

\section{1. csQCA Procedure}

First, the calibration process transformed all the data into either a $0=$ the outcome is not present, or $1=$ the outcome is present. From this newly calibrated data a truth table was created finding all the relevant conditions for $Y(0 ; 1)$ (as the dataset and truth tables were too large to fit within this paper, they are available upon request). Conforming with previous QCA research, we used the threshold of 0.90 for consistency in order to reduce the likelihood of true logical contradictions within the cases found. In line with recent QCA research, we too also implement the use of the parsimonious solution as this has been shown to be the best minimization technique for causal inference [50].

\subsection{SVM Procedure}

First of all, the 10-fold cross validation technique was used for the dataset, therefore, $10 \%$ of the given dataset was for testing and $90 \%$ was for training sets [68]. Second, SVM classifiers were constructed using polynomial, RBF and Pearson VII universal function (PUF) kernels. In addition, Bagging (BA), Random Subspace (RSS) and AdaBoost (ADA) were also used as ensemble classifiers of the SVM model. Lastly, accuracy, the F-score and AUC were used to observe the performance of the SVM models from a diverse perspective.

\subsection{Dataset}

The dataset for this study was conducted through a professional online survey company from March 13 to March 16, 2018. The sample used a proportional allocation extraction method based on age and gender by using the online surveyor's panel. The final samples of the survey consisted of 339 people who are in their 20 50s, have experience in using the airport in the past but did not experience the biometric boarding system. The demographic composition of the samples is shown in Table 2. The questionnaire used in the dataset of this study was measured on a Likert five-point scale ("totally disagree" to "totally agree"). The questionnaires are shown in Table 3.

Table 2. Demographic information of the dataset.

\begin{tabular}{cccc}
\hline Variables & Type & $\mathbf{N}$ & $\mathbf{\%}$ \\
\hline \multirow{2}{*}{ Gender } & Male & 168 & 49.6 \\
& Female & 171 & 50.4 \\
Age & $20 \mathrm{~s}$ & 84 & 24.8 \\
& $30 \mathrm{~s}$ & 84 & 24.8 \\
& $40 \mathrm{~s}$ & 84 & 24.8 \\
Frequency of Airport Use & $50 \mathrm{~s}$ & 87 & 25.7 \\
& 1 3 Times & 297 & 87.6 \\
& 4 6 Times & 30 & 8.8 \\
& 6 9 Times & 6 & 1.8 \\
& Over 10 Times & 6 & 1.8 \\
\hline
\end{tabular}

\subsection{Constructing Classifiers}

In order to test our dataset, the open source tool WEKA (https://www.cs.waikato.ac.nz/ml/index. $\mathrm{html}$ ) on a Macintosh with a 3.2GHz Intel Core i7 CPU and 32GB 2400MHz DDR4 RAM was utilized. Three separate tests were implemented so that all three dependent variables (Weak Resistance and Strong Resistance) could be individually tested based on the identified independent variables. Within these three separate tests we explored SVM classifiers with polynomial, RBF and Pearson VII universal function (PUF) kernels. Consequently, comparisons between single SVM classifiers and ensemble SVM classifiers such as BA, RSS and ADA were performed. As we were dealing with a class imbalance and a multiclass problem, traditional binary classification metrics were not enough to serve a true 
representation of our results. Thus, we implemented the use of three metrics that are better suited to cope with these issues, these being accuracy, F-measure and ROC.

Table 3. Survey questions of the dataset.

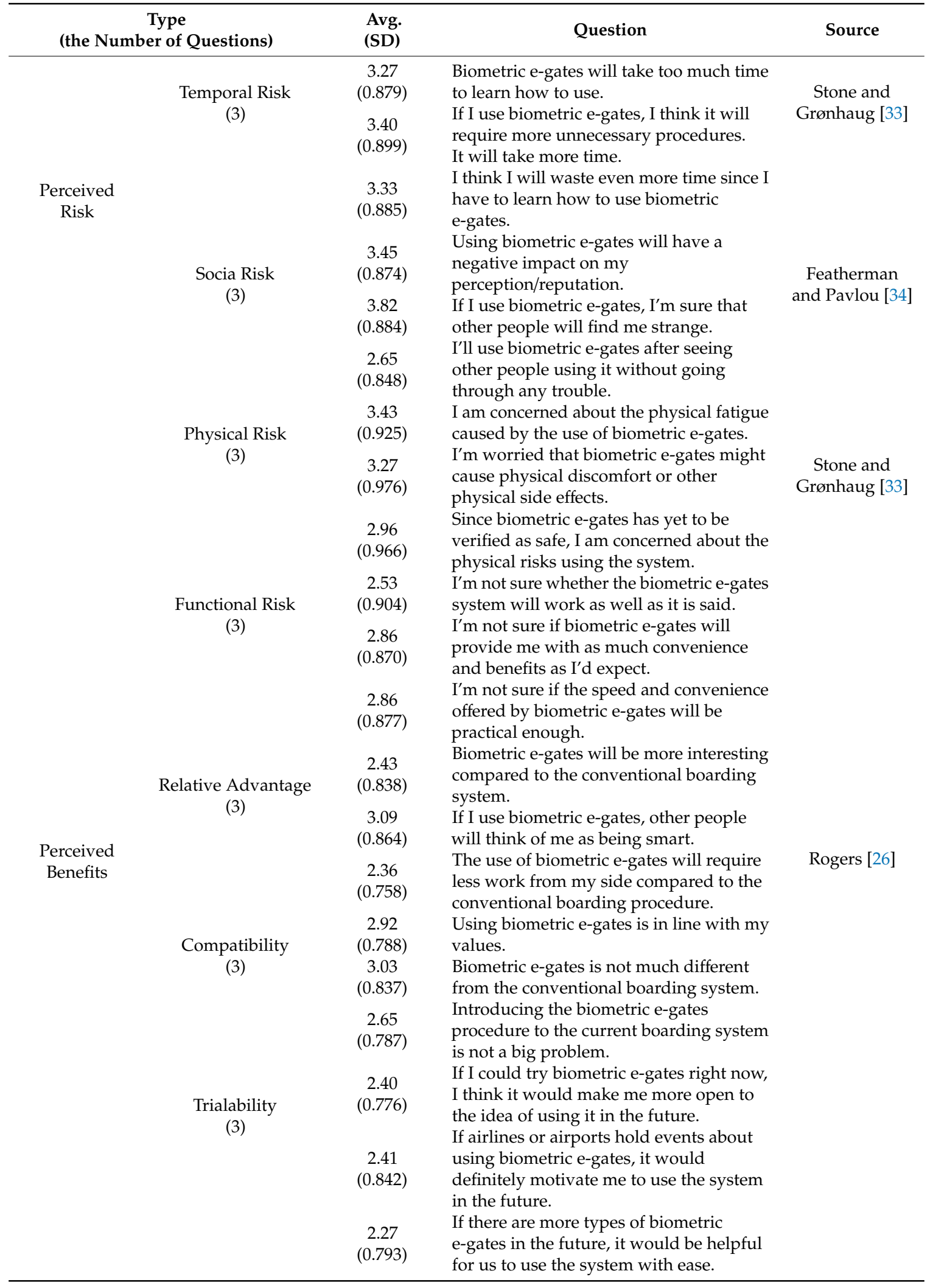


Table 3. Cont.

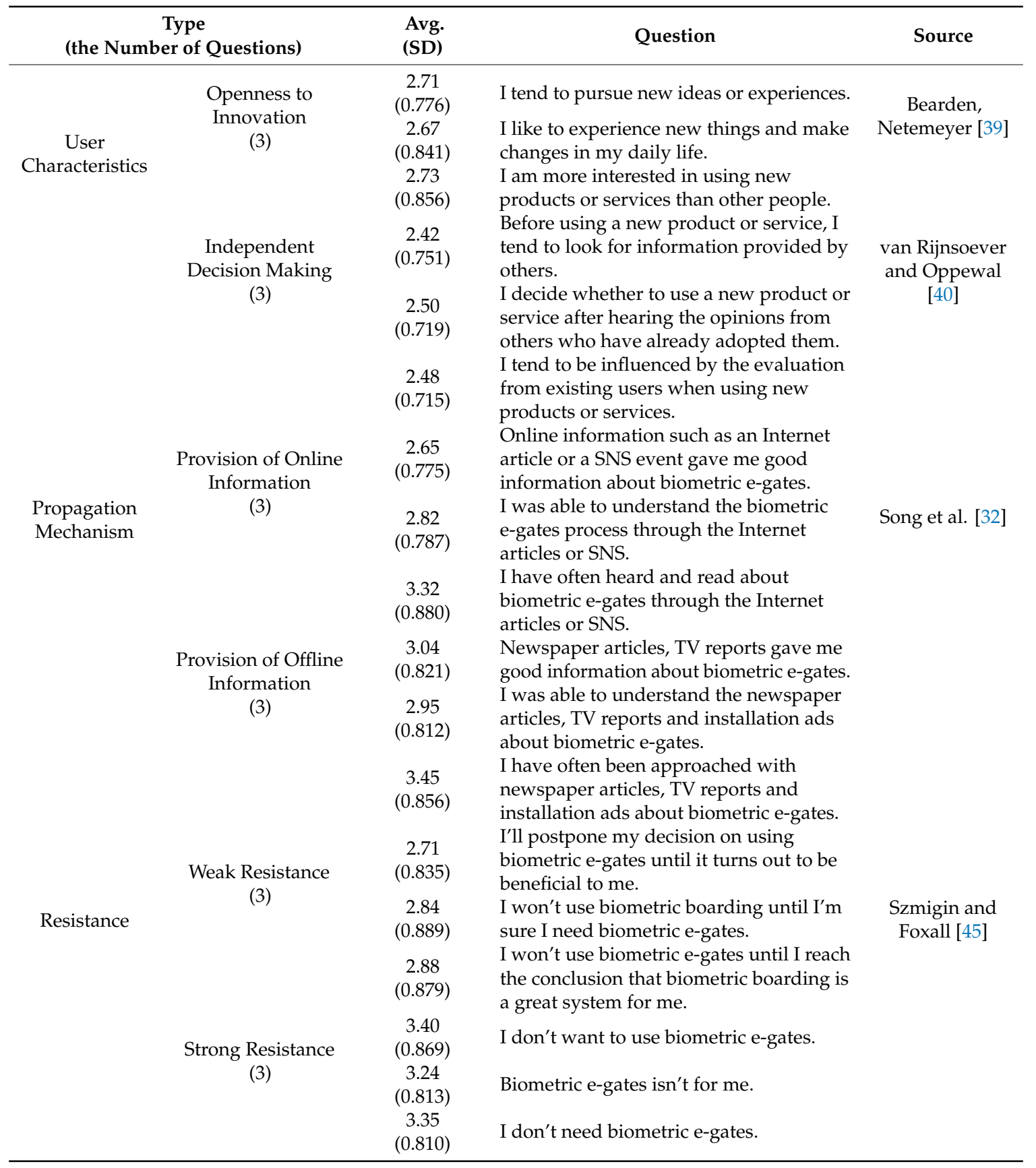

\section{Results}

\section{1. $\operatorname{csQCA}$}

To begin with, we performed necessity tests on both the dependent variables in order to find out the necessary conditions. Before approaching necessity tests, one must be careful to not overestimate the ability of the computer to find necessity relations. Duşa advices "It is the researcher who should make sense out of all these necessity relations and select only those which have a theoretical meaning" [47] (p.122). By looking at Table 4, the first test based on the presence of WR, Models 1 and 4 show no relation to prior literature and thus we reject these necessity conditions. Models 2 and 3 however show potential conditions. First, Model 2 claims that the presence of PR OR UC is necessary to explain WR. Furthermore, Model 3 claims that the presence of PR OR PM is necessary to explain WR. It is clear that 
the presence of PR is the common factor present, however as it is within two models where the factors UC and PM could be the factors present, we have to negate the outcome to see if any further conditions appear that add rigor to this claim. As can be seen in the results, when we make the outcome no resistance, the condition whereby $\sim \mathrm{PR}+\mathrm{PB}$ is found. This model backs up the previous literature as it is clear that in order to have no Weak Resistance you need to have no perceived risks or perceived benefits. Thus, we conclude that PR is a necessary condition of WR.

Table 4. Analysis of necessary conditions on resistance.

\begin{tabular}{ccccc}
\hline Model & & incIN * & RoN $^{* *}$ & $\operatorname{covN}^{* * *}$ \\
\hline & $\sim \mathrm{PR}+\sim \mathrm{PB}$ & 0.921 & 0.208 & 0.670 \\
Weak Resistance is Present (WR) & $\mathrm{PR}+\mathrm{UC}$ & 0.930 & 0.211 & 0.676 \\
& $\mathrm{PR}+\mathrm{PM}$ & 0.921 & 0.246 & 0.681 \\
Weak Resistance is not present $(\sim \mathrm{WR})$ & $\mathrm{UC}+\sim \mathrm{PM}$ & 0.921 & 0.208 & 0.670 \\
& $\sim \mathrm{PR}+\mathrm{PB}$ & 0.955 & 0.224 & 0.373 \\
Strong Resistance is present (SR) & $\sim \mathrm{PR}+\sim \mathrm{PM}$ & 0.902 & 0.231 & 0.356 \\
& $\sim \mathrm{PR}+\sim \mathrm{PB}$ & 0.922 & 0.491 & 0.910 \\
& $\mathrm{PR}+\mathrm{UC}$ & 0.922 & 0.491 & 0.910 \\
Strong Resistance is not present $(\sim \mathrm{SR})$ & $\mathrm{PR}+\mathrm{PM}$ & 0.916 & 0.561 & 0.919 \\
& $\mathrm{UC}+\sim \mathrm{PM}$ & 0.919 & 0.482 & 0.907 \\
& $\mathrm{~PB}+\sim \mathrm{UC}+\sim \mathrm{PM}$ & 0.903 & 0.273 & 0.110 \\
\hline
\end{tabular}

${ }^{*}$ Coverage; ${ }^{* *}$ Relevance of Necessity; ${ }^{* * *}$ Raw Coverage

The next test which was made on the dependent variable SR shows five possible models. Models 1,3 and 5 do not have any prior theoretical explanation and thus we reject these necessity conditions. Models 2 and 3 shows the exact same conditions as seen within the WR dependent variable test and therefore we conclude the same explanation for this outcome as was made in test one. Once again in order to clarify the potential necessity of PR on SR we once again negated the outcome to see what factors were necessary. Two models were presented and first we rejected Model 2 as this model does not have any theoretical rigor based on prior literature. Next, and more importantly, we can see that $\sim \mathrm{PR}$ is a necessary condition to explain $\sim \mathrm{SR}$. In layman's terms, this shows that when there is no presence of SR, there is also no presence of PR. This condition conforms to prior literature and as this variable is alone in its condition, we can strongly affirm that PR is a necessary condition of the outcome SR.

Next we undertook sufficiency tests. As can be seen in Table 5, Weak Resistance is represented in two constellations combined by the logic AND function $(+)$ creating an overall model consistency of 0.957. If we look at the model it explains that the presence of PR is a sufficient condition to explain Weak Resistance. In the first configuration, the presence of PR alongside the absence of PB and UC shows Weak Resistance with a consistency of 1 . The next configuration shows that the presence of PR and the absence of PB and PM is a sufficient condition for Weak Resistance. Notably, in both configurations we can see the presence of $P R$ and absence of $P B$, suggesting these are the main constructs that affect Weak Resistance. 
Table 5. Analysis of sufficient conditions on resistance.

\begin{tabular}{|c|c|c|c|c|c|c|c|}
\hline \multirow{3}{*}{ Construct } & \multicolumn{2}{|c|}{ Weak Resistance } & \multicolumn{5}{|c|}{ Strong Resistance } \\
\hline & \multicolumn{7}{|c|}{ Configuration } \\
\hline & 1 & 2 & 1 & 2 & 3 & 4 & 5 \\
\hline PR & $\bullet$ & $\bullet$ & $\bullet$ & $\bullet$ & & $x$ & \\
\hline PB & $x$ & $x$ & $x$ & & • & - & $x$ \\
\hline UC & $x$ & & & & $x$ & & - \\
\hline PM & & $x$ & & $\bullet$ & & $x$ & $\bullet$ \\
\hline Consistency & 1.000 & 0.952 & 1.000 & 0.964 & 0.952 & 1.000 & 0.965 \\
\hline PRI & 1.000 & 0.952 & 1.000 & 0.964 & 0.952 & 1.000 & 0.965 \\
\hline Raw Coverage & 0.026 & 0.088 & 0.169 & 0.172 & 0.065 & 0.032 & 0.266 \\
\hline Unique coverage & 0.009 & 0.070 & 0.068 & 0.062 & 0.049 & 0.026 & 0.172 \\
\hline Overall solution consistency & \multicolumn{2}{|c|}{0.957} & \multicolumn{5}{|c|}{0.962} \\
\hline Overall solution coverage & \multicolumn{2}{|c|}{0.097} & \multicolumn{5}{|c|}{0.494} \\
\hline
\end{tabular}

For Strong Resistance, the configurations are slightly more complex. Based on the raw coverage, we can see that Configurations 1, 2 and 5 are the most prominent for explaining Strong Resistance, and therefore our focus will be put on explaining these. The first configuration conforms with the results from Weak Resistance whereby PR is present and PB is absent, suggesting that once again these two constructs when in this state have sufficiency in explaining Strong Resistance. Next, Configuration 2 once again shows that PR is present. However, interestingly, it shows that this is when PM is present too. This suggests that online information can potentially have a positive impact on the resistance of biometric e-gate technology adoption. Lastly, the most interesting outcome was seen in Configuration 5. First, it is clear that the absence of PB has an impact on Strong Resistance conforming to prior literature and our own hypothesis. However, the csQCA analysis also states that no PB OR UC OR PM is a sufficient condition. This could be explained through the notion that people who have an openness to new technology or to trying new innovations will negatively react to an innovation in the presence of perceiving no benefits. With this we argue that this can be because the feeling of no PB outweighs the feelings seen within UC and PM, and thus the outcome would be Strong Resistance as expectations would be outweighed by disappointment. Both models presented to explain both types of resistance can be visualized in Figure 1, whereby the red color represents instances of resistance as the output condition based on the sufficiency of the areas of each construct.

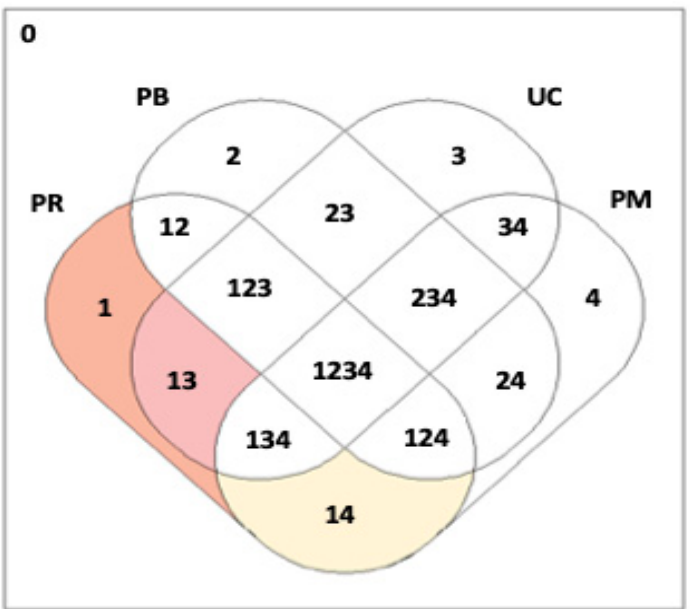

(a)

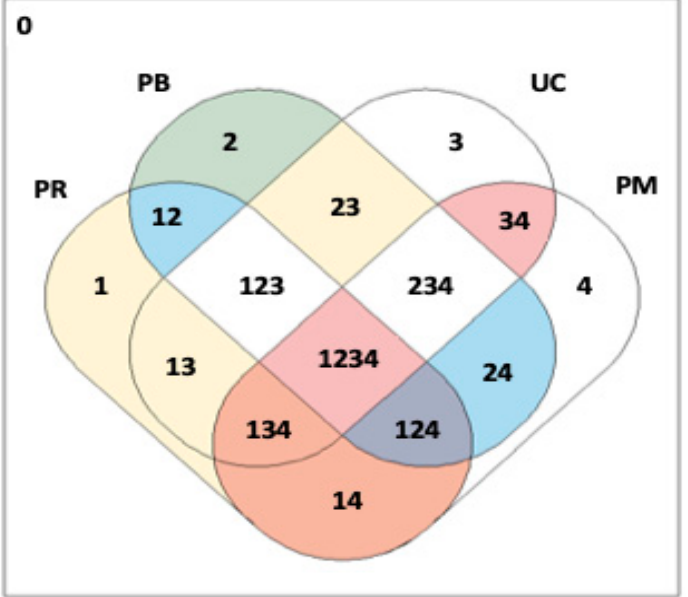

(b)

Figure 1. Venn diagrams of sufficient conditions for Weak (a) and Strong (b) Resistance. 


\subsection{Single SVM Classifiers}

Figure 2and Figure 3 indicate the evaluation results of performances of the single SVM classifiers with three different kernel functions (polynomial, RBF and PUF) on the given dataset. The evaluation of each performance was measured in accuracy, F-score and AUC.

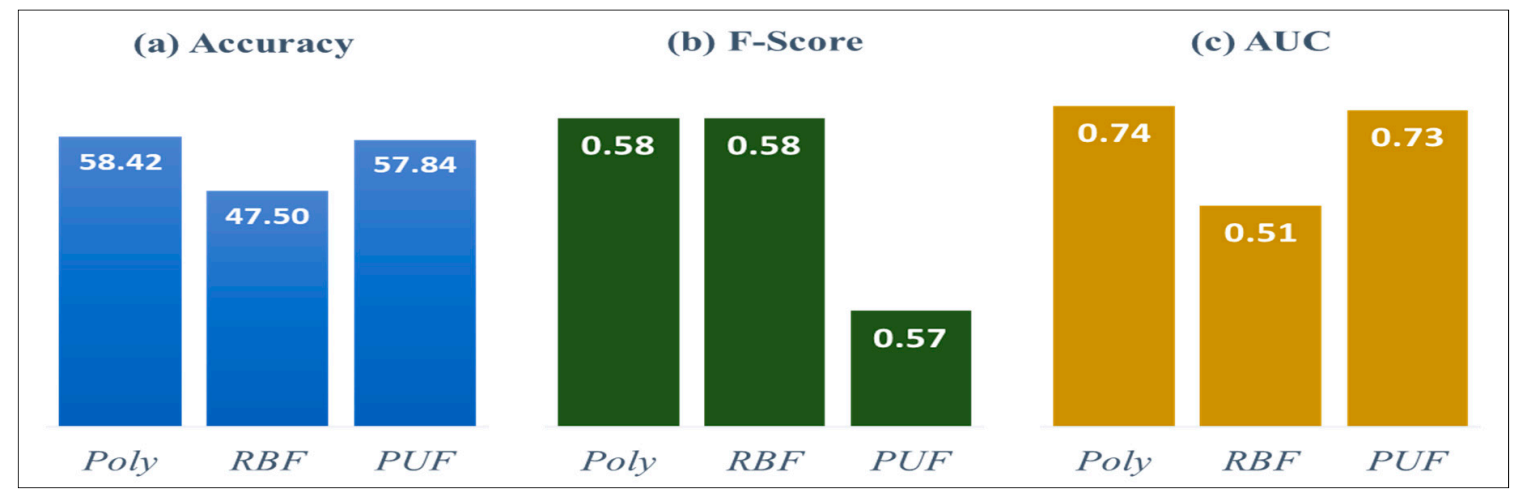

Figure 2. Performance of single SVM classifiers for Weak Resistance based on accuracy (a), F-Score (b) and AUC (c).

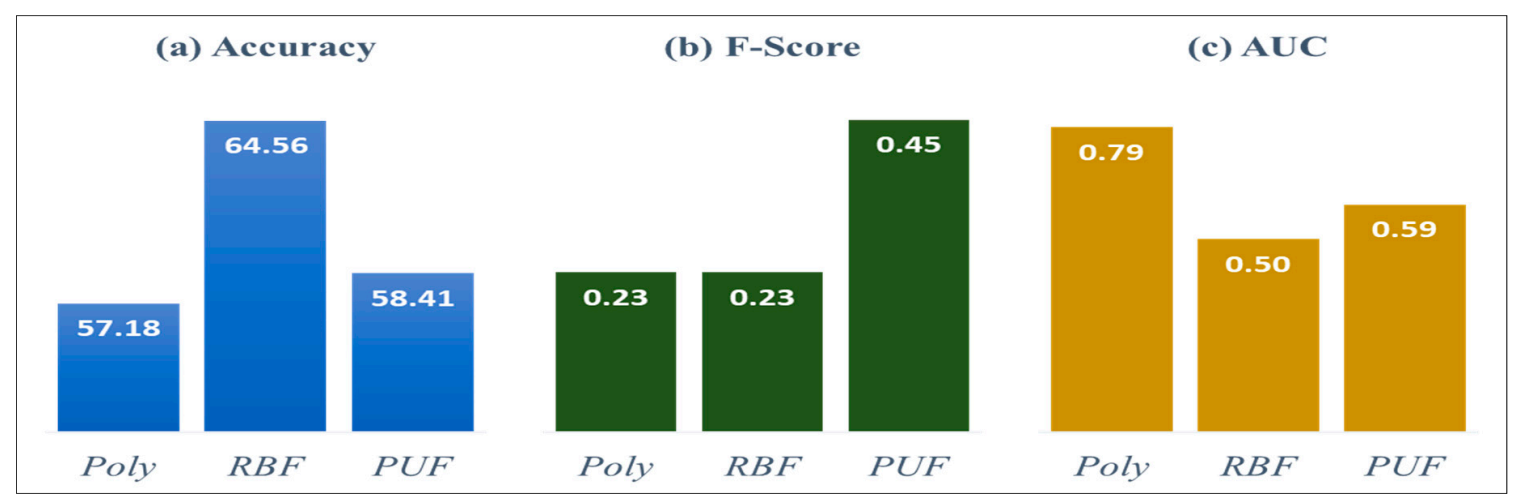

Figure 3. Performance of single SVM classifiers for Strong Resistance based on accuracy (a), F-Score (b) and AUC (c).

As Figure 2 shows the performance evaluation for the dependent variable Weak Resistance, the polynomial kernel with an SVM classifier has a better performance than the others in terms of accuracy (58.42\%). In addition, in terms of F-score, polynomial and RBF kernels had the best score (0.58), while the polynomial kernel acquired the best (0.74) for performance evaluation of AUC.

For the dependent variable Strong Resistance in Figure 3, contrary to the performance evaluation seen in the dependent variable Weak Resistance, the result shows that RBF calculates the best result $(64.56 \%)$ in terms of accuracy. As is shown in Table 6, a single SVM classifier with a PUF kernel makes the best score (0.45) for the F-score. Nevertheless, the polynomial kernel has the best results in AUC performance evaluation (0.79)

Table 6. Best performance for single SVM classifiers.

\begin{tabular}{cccc}
\hline Dependent Variable & Accuracy & F-Score & AUC \\
\hline Weak Resistance & Polynomial & Polynomial, RBF & Polynomial \\
Strong Resistance & RBF & PUF & Polynomial \\
\hline
\end{tabular}




\subsection{Ensemble SVM Classifiers}

Figures 4 and 5 represent the performances of the ensemble SVM classifiers with Bagging. In addition, the models are processed with polynomial, RBF and PUF kernels in the given dataset. As the first experiment with single SVM classifier models, accuracy, F-score and AUC were measured.

Figure 4 shows BA with an SVM classifier, where the combination with the PUF function acquires the best performance results in accuracy $(58.72 \%)$ and AUC (0.77) for the target variable Weak Resistance. On the other hand, polynomial and RBF obtain better performance measurement in the F-score (0.58).

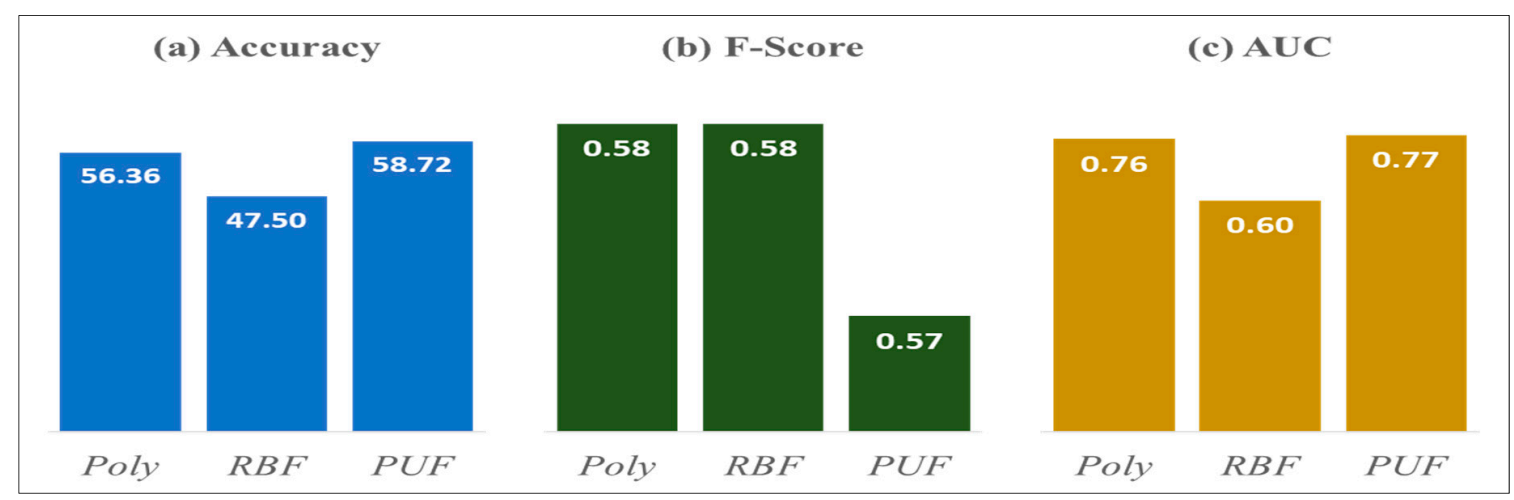

Figure 4. Performance of an ensemble SVM classifier (BA) for Weak Resistance based on accuracy (a), F-Score (b) and AUC (c).

For the dependent variable Strong Resistance in Figure 5, the performance with a single kernel function can be found for the SVM classifier with Bagging: RBF in accuracy (65.75\%), PUF in F-score $(0.50)$ and polynomial in AUC (0.86) show the best performance, respectively. Table 7 addresses best performance for ensemble SVM (BA).

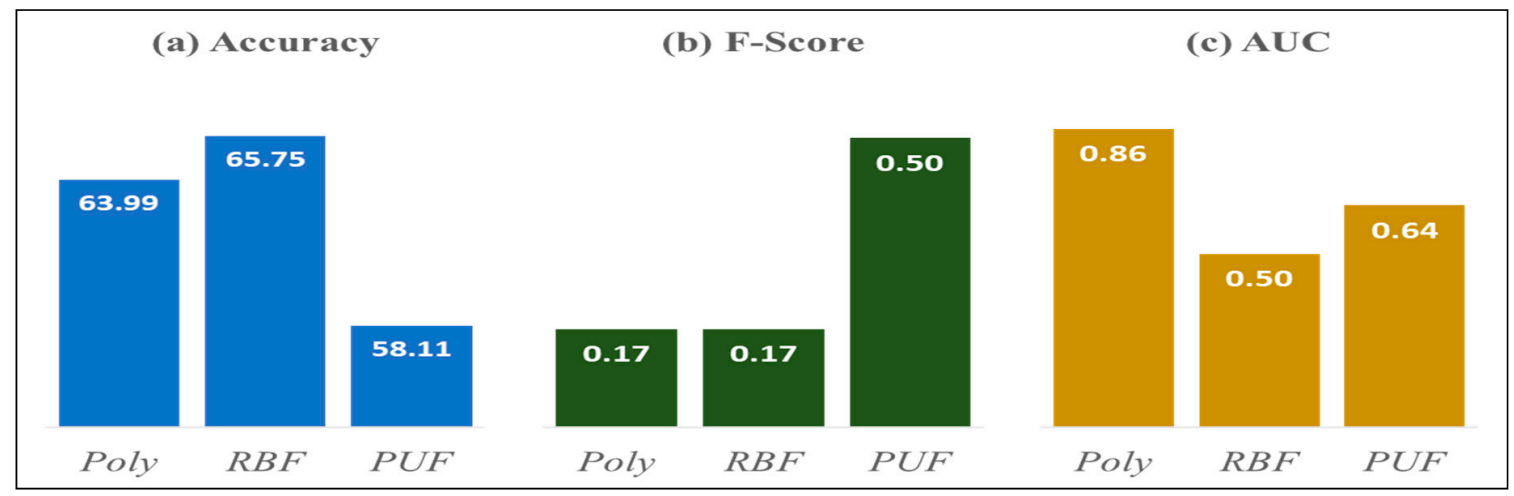

Figure 5. Performance of an ensemble SVM classifier (BA) for Strong Resistance based on accuracy (a), F-Score (b) and AUC (c).

Table 7. Best Performance for ensemble SVM classifiers (BA).

\begin{tabular}{cccc}
\hline Dependent Variable & Accuracy & F-Score & AUC \\
\hline Weak Resistance & PUF & Polynomial, RBF & PUF \\
Strong Resistance & RBF & PUF & Polynomial \\
\hline
\end{tabular}

The performance result in Figure 6, and the result of PUF with RSS SVM derives the best score in all performance metrics for Weak Resistance: $57.25 \%$ in accuracy, 0.51 in F-score and 0.75 in AUC, respectively.

For the dependent variable Strong Resistance in Figure 7, using an RBF kernel with RSS ensemble SVM models shows the superior performance in accuracy (65.16\%). However, polynomial and RBF 
cannot be used in terms of F-score, since their datasets were overfitted, while the PUF kernel has the highest score (0.40). In terms of AUC, the polynomial kernel have a relatively better performance than other kernels (0.86). Table 8 summarizes the best performance for ensemble SVM classifiers (RSS).

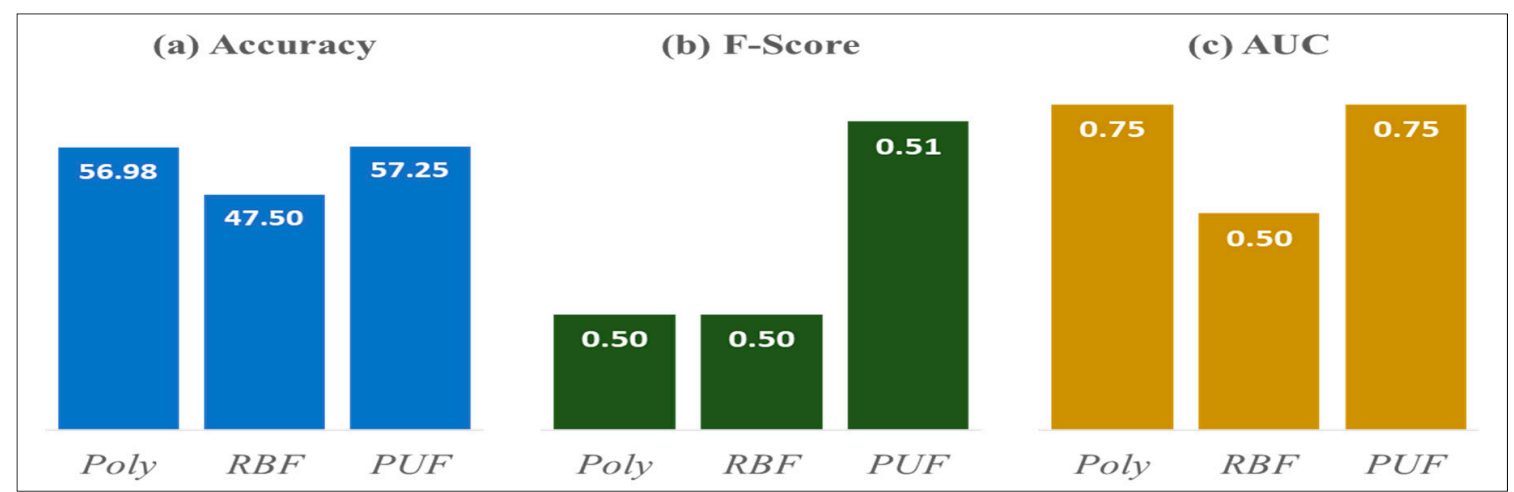

Figure 6. Performance of an ensemble SVM classifier (RSS) for Weak Resistance based on accuracy (a), F-Score (b) and AUC (c).

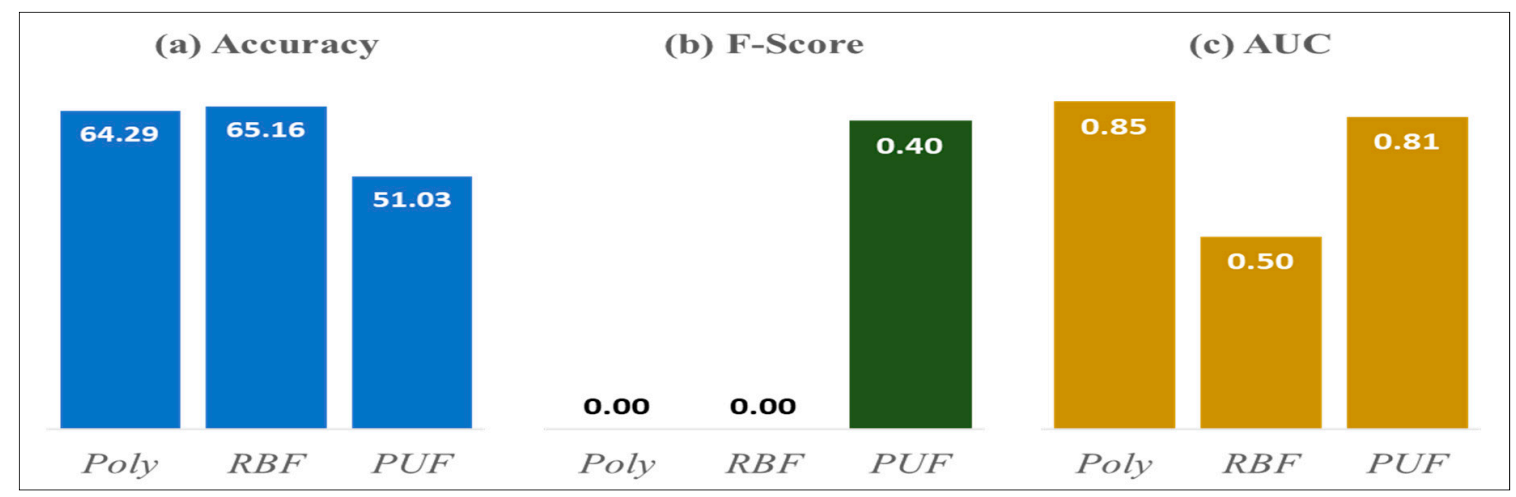

Figure 7. Performance of an ensemble SVM classifier (RSS) for Strong Resistance based on accuracy (a), F-Score (b) and AUC (c).

Table 8. Best performance for ensemble SVM classifiers (RSS)

\begin{tabular}{cccc}
\hline Dependent Variable & Accuracy & F-Score & AUC \\
\hline Weak Resistance & PUF & PUF & Polynomial, PUF \\
Strong Resistance & RBF & PUF & Polynomial \\
\hline
\end{tabular}

Figures 8 and 9 reveal the performances of the ADA ensemble SVM classifiers with three different kernel functions (polynomial, RBF and PUF) in terms of accuracy, F-score and AUC of the given dataset. As Figure 7 shows, the polynomial kernel has the highest performance result (58.42\%) compared with RBF and PUF kernels with ADA ensemble SVM classifiers in accuracy for the dependent variable Weak Resistance. In terms of the F-score, polynomial and RBF both have the best performance (0.58), while, interestingly, PUF acquires superior performance (0.74) compared with other kernels in AUC evaluation.

For the dependent variable Strong Resistance in Figure 8, RBF presents relatively better performance in accuracy $(64.56 \%)$ and F-score (0.30) performance measurement while PUF shows the best AUC performance (0.76) with ADA ensemble SVM models. Table 9 also states the best performance for ensemble SVM classifiers (ADA). 


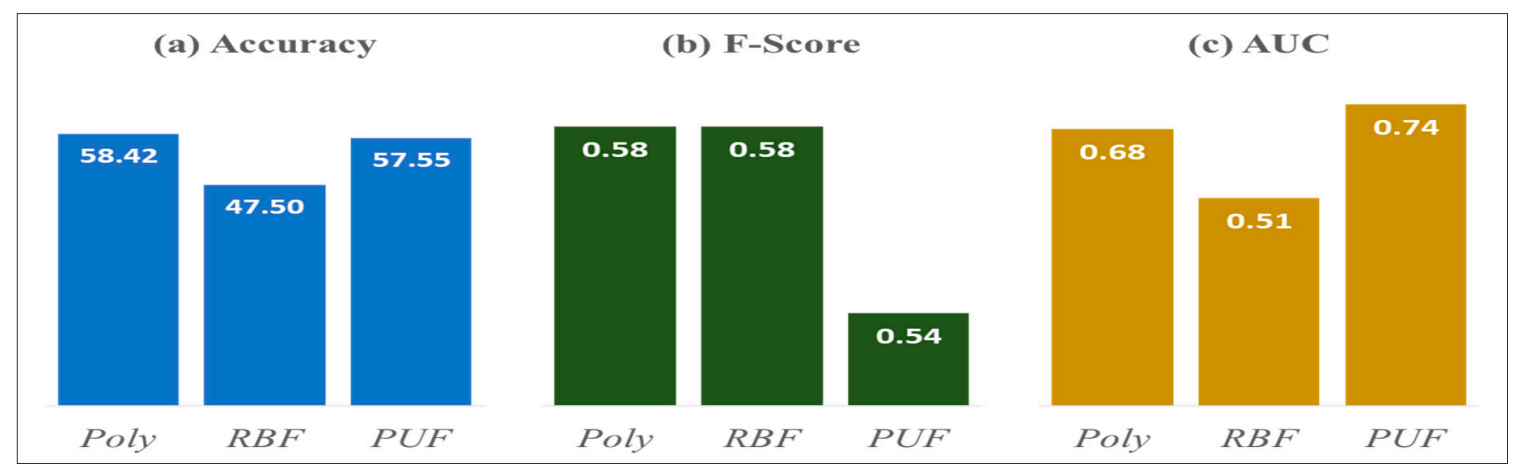

Figure 8. Performance of an ensemble SVM classifier (ADA) for Weak Resistance based on accuracy (a), F-Score (b) and AUC (c).

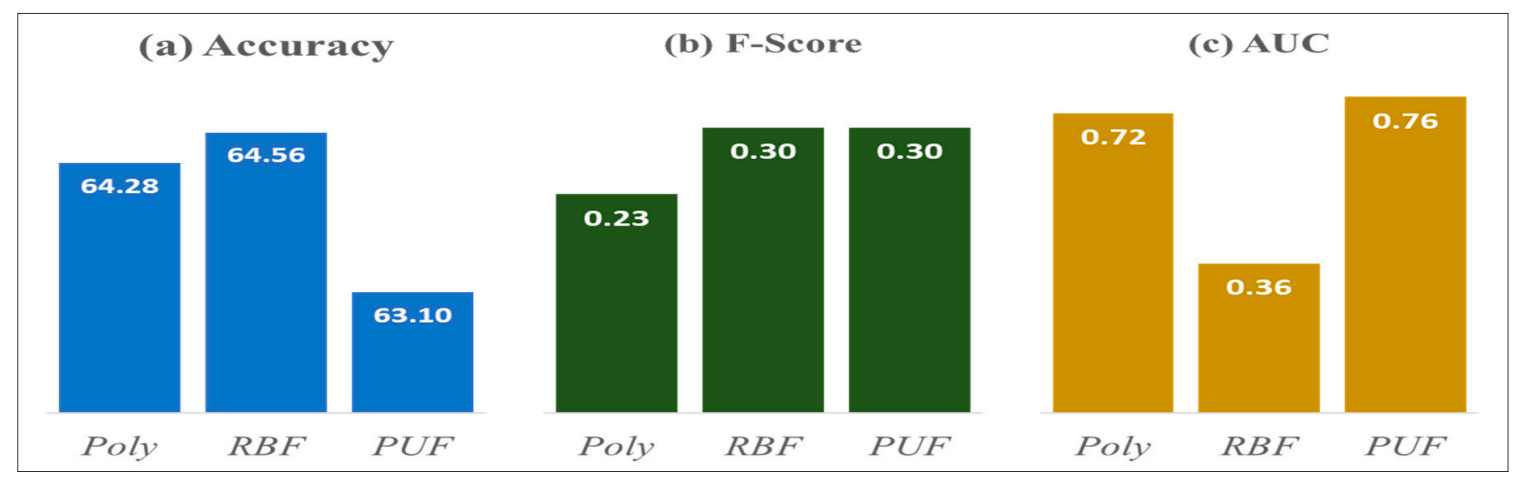

Figure 9. Performance of an ensemble SVM classifier (ADA) for Strong Resistance based on accuracy (a), F-Score (b) and AUC (c).

Table 9. Best performance for ensemble SVM classifiers (ADA).

\begin{tabular}{cccc}
\hline Dependent Variable & Accuracy & F-Score & AUC \\
\hline Weak Resistance & Polynomial & Polynomial, RBF & PUF \\
Strong Resistance & RBF & RBF, PUF & PUF \\
\hline
\end{tabular}

Below Table 10 shows overall results of SVM classifier performance evaluations for each dependent variable (Weak Resistance and Strong Resistance) that were used in this research.

Table 10. The best performance results of SVM classifiers for each dependent variable.

\begin{tabular}{ccccc}
\hline Weak Resistance & Single SVM & BA SVM & RSS SVM & ADA SVM \\
\hline \multirow{2}{*}{ Accuracy } & Polynomial & PUF & PUF & Polynomial \\
& $58.42^{*} \%$ & $58.7 \%^{*}$ & $57.25 \%$ & $58.42 \%$ \\
F-Score & Polynomial, RBF & Polynomial, RBF & PUF & Polynomial, RBF \\
& $0.58^{*}$ & $0.58^{*}$ & 0.51 & $0.58^{*}$ \\
AUC & Polynomial & PUF & Polynomial, PUF & PUF \\
& 0.74 & $0.77^{*}$ & 0.75 & 0.74 \\
\hline Strong Resistance & Single SVM & BA SVM & RSS SVM & ADA SVM \\
\hline \multirow{2}{*}{ Accuracy } & RBF & RBF & RBF & RBF \\
& $64.56 \%$ & $65.75 \% *$ & $65.16 \%$ & $64.56 \%$ \\
F-Score & PUF & PUF & PUF & RBF, PUF \\
& 0.45 & $0.50^{*}$ & 0.40 & 0.30 \\
AUC & Polynomial & Polynomial & Polynomial & PUF \\
& 0.79 & $0.86^{*}$ & 0.85 & 0.76 \\
\hline
\end{tabular}

* The highest score in row 


\section{Discussion}

\subsection{Key Findings}

This study looked to explore the reasons why flight passengers are reluctant to adopt the biometric e-gate boarding system. The sustainability of airport operations relies on the airports' ability to adapt to using more advanced, non-invasive and reliable technologies that can provide a satisfactory passenger service for generations to come. However, these technologies do not always provide the best solution. This paper has attempted to explore one such example of where a failed attempt to increase passenger service quality has led to managers looking for alternative solutions that can help to aid the sustainability of service quality despite the ever-increasing demands made through passenger numbers. Through an extensive self-report survey effort, 336 valid questionnaire samples were garnered and two types of resistance to the adoption of innovative technology, like biometric e-gate security, were analyzed in the form of Weak Resistance and Strong Resistance.

Our first key findings within this paper was using csQCA [29]. With csQCA we were able to find two sufficient configurations that explain Weak Resistance and three configurations that explain Strong Resistance to the biometric e-gate security system. Furthermore, all these configurations fit within the commonly used threshold of 0.90 [34]. Weak Resistance was firstly explained through (1) perceived risks * no perceived benefits * low user characteristics and (2) perceived risks * no perceived benefits * low propagation mechanisms. For Strong Resistance, the following three configurations were found: (1) perceived risks * no perceived benefits, (2) perceived risks * high propagation mechanism and (3) no perceived benefits * high user characteristics * high propagation mechanism. The overall findings from these configurations show that presence of perceived risks and absence of perceived benefits were the overarching key issues promoting resistance to air passengers from using the biometric e-gate technology. Therefore, future implementation of these services will have to take into consideration these factors in order to increase the chances of a successful implementation. Interestingly, the inclusion of propagation mechanisms was also seen in both dependent variables and therefore online information was seen to have an impact on the resistance to the technology. This means that managers should take into consideration online presence as well as the risks and benefits associated with the use of biometric e-gate services.

With csQCA one can find patterns within data and make decisions based on these findings, however it lacks the power to make quick and fast predictions based on the data. Therefore, we next wanted to explore the power of a classification technique that would help managers quickly assess the implementation of new technologies within the airport setting, including our case of biometric e-gate technology. Starting with the first dependent variable which attempted to predict people's feelings on Weak Resistance. We found that a Bagging algorithm with a PUF kernel SVM model yields the best overall model with an accuracy of $58.72 \%$, F-score of 0.57 and AUC of 0.77 . Overall, in our test of this dependent variable, it is safe to presume that the best performance of the models lies with the use of BA in most cases. Another point to note about this test is that the overall level of accuracy and F-score is fairly low, proving this task to be a challenging one in the machine-learning domain. Another key outcome of this test was seen in the F-score by RSS SVM polynomial and RBF with the score of 0.0 meaning that the model was overfit.

Next, if we look at the second dependent variable Strong Resistance, the best accuracy was seen within the BA SVM RBF kernel model. F-score evaluation shows the best score with a BA SVM PUF of 0.50 . However, as previously stated, due to the type of problem we are trying to learn with these models, the F-score is not reliable enough on its own. However, the AUC score presented by the BA SVM polynomial with 0.86 is reliable enough to compare to the result of the F-score. Once again, an ensemble model has been the best performer in this test compared to single SVM models. The overall performance of the BA SVM model shows more confidence in these models' ability to predict the target variable (Strong Resistance) and yield greater reliability compared to the model's usage seen in the dependent variable Weak Resistance. 


\subsection{Theoretical Implications}

Theoretically, this paper has the following implications. First, this paper enriches the innovation adoption field of research by exploring resistance to innovation. This allows for a reverse analysis of the reasons people adopt innovation and thus can provide unique implications based on the results [27]. The context of biometric e-gate technology provides a case in which complex decisions have to be made by passengers who are entrenched within an old security system that has been in place for decades at airports. However, these systems are slowly coming to capacity and thus their sustainability is in question. With our analysis we have used an SVM classification methodology which can help managers quickly assess the resistance to biometric e-gate technology. Secondly we have shown how they can successfully determine what the problems are based on the use of csQCA, which allows for the identification of "multiple conjunctural causation" [48].

\subsection{Limitations and Future Research}

This study has limitations. First, this research was not able to measure the detailed experience of the consumers' past encounters with biometric-related services. Considering the fact that biometric identification is already being used in various areas such as building entries, mobile payment and ATM authentication, it will also be meaningful to test the performance of this prediction model for resistance against similar services from other fields. In particular, analyzing the case where the usage rate of the "Automated Immigration Service of Korea" using fingerprints have risen from $24.8 \%$ in 2014 to $43.8 \%$ in 2017 [69] may let us find an important issue that this research was not able to consider for another sustainability issue at airports. Therefore, it is necessary to carry out a study comparing performance of SVM prediction models on resistance in the adoption process of various innovative products and services that utilizes biometric recognition.

Author Contributions: Conceptualization, K.C.L. and C.K.; methodology, K.C.L. and C.K.; software, F.J.C. and C.K.; validation, C.K. and F.J.C.; formal analysis, C.K. and F.J.C.; investigation, C.K., F.J.C. and K.C.L.; data curation, C.K.; writing-original draft preparation, C.K. and F.J.C; writing-review and editing, K.C.L.; supervision, K.C.L.

Funding: The APC was funded by BK21 project by the government of Korea.

Conflicts of Interest: The authors declare no conflict of interest.

\section{References}

1. Park, J.-W.; Ryu, Y.K. Investigating the Effects of Airport Servicescape on Airport Users' Behavioral Intentions: A Case Study of Incheon International Airport Terminal 2 (T2). Sustainability 2019, 11, 4171. [CrossRef]

2. Wensveen, J. Air Transportation: A Management Perspective; Routledge: London, UK, 2018.

3. Xu, X.; Zhou, Z.; Dou, Y.; Tan, Y.; Liao, T. Sustainable Queuing-Network Design for Airport Security Based on the Monte Carlo Method. Sustainability 2018, 10, 1. [CrossRef]

4. Morosan, C. An empirical examination of US travelers' intentions to use biometric e-gates in airports. J. Air Trans. Manag. 2016, 55, 120-128. [CrossRef]

5. Ali, A.B. Travel and Trourism Management; Delhi: PHI Learning Private Limited: Delhi, India, 2015.

6. Kim, M.W. Social Responsibility Report 2018; Korea Airports Corporation: Seoul, Korea, 2018.

7. Marx, A.; Rihoux, B.; Ragin, C. The origins, development, and application of Qualitative Comparative Analysis: The first 25 years. Eur. Political Sci. Rev. 2014, 6, 115-142. [CrossRef]

8. Zu, X.; Wu, Y.; Zhang, Z.; Yu, L. Prediction of Consumption Choices of Low-Income Groups in a Mixed-Income Community Using a Support Vector Machine Method. Sustainability 2019, 11, 3981. [CrossRef]

9. Alimi, O.A.; Ouahada, K.; Abu-Mahfouz, A.M. Real Time Security Assessment of the Power System Using a Hybrid Support Vector Machine and Multilayer Perceptron Neural Network Algorithms. Sustainability 2019, 11, 3586. [CrossRef]

10. Xue, Y.; Ren, J.; Bi, X. Impact of Influencing Factors on CO2 Emissions in the Yangtze River Delta during Urbanization. Sustainability 2019, 11, 4183. [CrossRef] 
11. Mrówczyńska, M.; Sztubecka, M.; Skiba, M.; Bazan-Krzywoszańska, A.; Bejga, P. The Use of Artificial Intelligence as a Tool Supporting Sustainable Development Local Policy. Sustainability 2019, 11, 4199. [CrossRef]

12. Olivares-Mercado, J.; Toscano-Medina, K.; Sánchez-Perez, G.; Hernandez-Suarez, A.; Perez-Meana, H.; Sandoval Orozco, A.L.; García Villalba, L.J. Early Fire Detection on Video Using LBP and Spread Ascending of Smoke. Sustainability 2019, 11, 3261. [CrossRef]

13. Huang, M.; Chen, C.; Lin, W.; Ke, S.; Tsai, C. SVM and SVM Ensembles in Breast Cancer Prediction. PLoS ONE 2017, 12, 1-14. [CrossRef]

14. Sasikala, B.S.; Biju, V.G.; Prashanth, C.M. Kappa and accuracy evaluations of machine learning classifiers. In Proceedings of the 2nd IEEE International Conference on Recent Trends in Electronics, Information \& Communication Technology, Bangalore, India, 19-20 May 2017.

15. Abdelaal, M.M.A.; Sena, H.A.; Farouq, M.W.; Salem, A.-B.M. Using data mining for assessing diagnosis of breast cancer. In Proceedings of the IEEE International Multiconference on Computer Science and Information Technology, Wisła, Poland, 18-20 October 2010; pp. 11-17.

16. Ahmad, L.G.; Eshlaghy, A.T.; Poorebrahimi, A.; Ebrahimi, M.; Razavi, A.R. Using three machine learning techniques for predicting breast cancer recurrence. J. Health Med. Inform. 2013, 4, 124.

17. Huang, C.L.; Liao, H.C.; Chen, M.C. Prediction model building and feature selection with support vector machines in breast cancer diagnosis. Expert Syst. Appl. 2008, 34, 578-587. [CrossRef]

18. Salama, G.I.; Abdelhalim, M.B.; Zeid, M.A.E. Experimental comparison of classifiers for breast cancer diagnosis. In Proceedings of the 2012 Seventh International Conference on Computer Engineering \& Systems (ICCES), Cairo, Egypt, 27-29 November 2012; pp. 180-185.

19. Senturk, Z.K.; Kara, R. Breast cancer diagnosis via data mining: Performance analysis of seven different algorithms. Comput. Sci. Eng. 2014, 4, 35-46. [CrossRef]

20. You, H.; Rumbe, G. Comparative study of classification techniques on breast cancer FNA biopsy data. Int. J. Artif. Intell. Interact. Multimed. 2010, 1, 6-13. [CrossRef]

21. Kittler, J.; Hatef, M.; Duin, R.P.W.; Matas, J. On combining classifiers. IEEE Trans. Pattern Anal. Mach. Intell. 1998, 20, 226-239. [CrossRef]

22. Blalock, G.; Kadiyali, V.; Simon, D.H. The Impact of Post-9/11 Airport Security Measures on the Demand for Air Travel. J. Law Econ. 2007, 50, 731-755. [CrossRef]

23. Farrell, S. How airports can fly to self-service biometrics. Biom. Technol. Today 2016, 2016, 5-7. [CrossRef]

24. Wayman, J.; Jain, A.; Maltoni, D.; Maio, D. An Introduction to Biometric Authentication Systems; Springer: London, UK, 2005.

25. Byun, S. Perceptions towards High-tech Service among its Early Adopters: In the Case of Fingerprint-enabled ATMs. Mark. Manag. Res. 2010, 15, 43-69.

26. Rogers, E.M. Diffusion of Innovations; Free Press: New York, NY, USA, 2003.

27. Sheth, J.N. Psychology of innovation resistance: The less developed concept. Res. Mark. 1981, 4, $273-283$.

28. Garner, S.J. Perceived risk and information sources in services purchasing. Mid-Atl. J. Bus. 1986, $24,49-58$.

29. Kaplan, L.B.; Szybillo, G.J.; Jacoby, J. Components of perceived risk in product purchase: A cross-validation. J. Appl. Psychol. 1974, 59, 287-291. [CrossRef]

30. Ko, H.; Jung, J.; Kim, J.; Shim, S.W. Cross-cultural differences in perceived risk of online shopping. J. Interact. Advert. 2004, 4, 20-29. [CrossRef]

31. Shimp, T.A.; Bearden, W.O. Warranty and other extrinsic cue effects on consumers' risk perceptions. J. Consum. Res. 1982, 9, 38-46. [CrossRef]

32. Song, H.Y.; Jung, J.M.; Kim, Y. Factors affecting postponement, rejection, and opposition to 3DTV adoption. Media Econ. Cult. 2013, 11, 7-50.

33. Stone, R.N.; Grønhaug, K. Perceived risk: Further considerations for the marketing discipline. Eur. J. Mark. 1993, 27, 39-50. [CrossRef]

34. Featherman, M.S.; Pavlou, P.A. Predicting e-services adoption: A perceived risk facets perspective. Int. J. Hum.-Comput. Stud. 2003, 59, 451-474. [CrossRef]

35. Moon, D.Y. Diffusion Factors of STEAM Education-Based on Rogers' Diffusion of Innovations. Theory-J. Korean Pract. Arts Educ. 2016, 29, 133-150. [CrossRef]

36. Mintrom, M. Policy Entrepreneurs and the Diffusion of Innovation. Am. J. Political Sci. 1997, 41, 738-770. [CrossRef] 
37. Rogers, E.M.; Shoemaker, F.F. Communication of Innovations: A Cross-Cultural Approach; Free Press: New York, NY, USA, 1971.

38. Ram, S. A model of innovation resistance. Adv. Consum. Res. 1987, 14, 208-212.

39. Bearden, W.O.; Netemeyer, R.G.; Teel, J.E. Measurement of consumer susceptibility to interpersonal influence. J. Consum. Res. 1989, 15, 473-481. [CrossRef]

40. van Rijnsoever, F.J.; Oppewal, H. Predicting early adoption of successive video player generations. Technol. Forecast. Soc. Chang. 2011, 79, 558-569. [CrossRef]

41. Fain, D.; Roberts, M.L. Technology vs. consumer behavior: The battle for the financial services customer. J. Direct Mark. 1997, 11, 44-54. [CrossRef]

42. Laukkanen, T.; Kiviniemi, V. The role of information in mobile banking resistance. Int. J. Bank Mark. 2010, 28, 372-388. [CrossRef]

43. Laukkanen, T.; Sinkkonen, S. Consumer resistance to internet banking: Postponers, opponents and rejectors. Int. J. Bank Mark. 2008, 26, 440-455. [CrossRef]

44. Haas, E.P. Back to the Future-The Use of Biometrics, Its Impact of Airport Security, and How This Technology Should Be Governed. J. Air Law Commer. 2004, 69, 459-489.

45. Szmigin, I.; Foxall, G. Three forms of innovation resistance: The case of retail payment methods. Technovation 1998, 18, 459-468. [CrossRef]

46. Ragin, C.C. The Comparative Method: Moving beyond Qualitative and Quantitative Strategies; Univercity of California Press: Berkeley, CA, USA, 2014.

47. Duşa, A. QCA with R: A Comprehensive Resource; Springer: Berlin, Germany, 2018.

48. Rihoux, B.; Ragin, C.C. Configurational Comparative Methods: Qualitative Comparative Analysis (QCA) and Related Techniques; Sage Publications: Thousand Oaks, CA, USA, 2008.

49. Schneider, C.Q.; Wagemann, C. Set-Theoretic Methods for the Social Sciences: A Guide to Qualitative Comparative Analysis; Cambridge University Press: Cambridge, UK, 2012.

50. Liu, H.; Subramanian, A.M.; Hang, C. In Search of the Perfect Match: A Configurational Approach to Technology Transfer in Singapore. IEEE Trans. Eng. Manag. 2019, 1-12. [CrossRef]

51. Byun, H.; Lee, S.W. A survey on pattern recognition applications of support vector machines. Int. J. Pattern Recognit. Artif. Intell. 2003, 17, 459-486. [CrossRef]

52. Tsai, C.F.; Cheng, K.C. Simple instance selection for bankruptcy prediction. Knowl.-Based Syst. 2012, 27, 333-342. [CrossRef]

53. Yekkehkhany, B.; Safari, A.; Homayouni, S.; Hasanlou, M. A comparison study of different kernel functions for SVM-based classification of multi-temporal polarimetry SAR data. Int. Arch. Photogram Rem. Sens. Spatial Inform. Sci. 2014, 2, W3. [CrossRef]

54. Das, S.R.; Das, K.; Mishra, D.; Shaw, K.; Mishra, S. An empirical comparison study on kernel based support vector machine for classification of gene expression data set. Procedia Eng. 2012, 38, 1340-1345. [CrossRef]

55. Buck, T.A.E.; Zhang, B. SVM kernels optimization: An example in yeast protein subcellular localization prediction. Project Report, School of Computer Science, Carnegie Mellon University, Pittsburgh, USA. Available online: https://pdfs.semanticscholar.org/0671/da2cf0d288d526a17aa2d7eddebe489429ff.pdf (accessed on 8 June 2018).

56. Mezghani, D.B.A.; Boujelbene, S.Z.; Ellouze, N. Evaluation of SVM kernels and conventional machine learning algorithms for speaker identification. Int. J. Hybrid Inform. Tech. 2010, 3, 23-34.

57. Baten, A.K.M.A.; Chang, B.C.H.; Halgamuge, S.K.; Li, J. Splice site identification using probabilistic parameters and SVM classification. BMC Bioinformatics 2006, 7, S15. [CrossRef]

58. Zhang, G.; Ge, H. Support vector machine with a Pearson VII function kernel for discriminating halophilic and non-halophilic proteins. Comput. Biol. Chem. 2013, 46, 16-22. [CrossRef]

59. Joshi, J.; Doshi, R.; Patel, J. Diagnosis and prognosis breast cancer using classification rules. International J. Eng. Res. Gen. Sci. 2014, 2, 315-323.

60. Aruna, S.; Rajagopalan, S.P.; Nandakishore, L.V. Knowledge based analysis of various statistical tools in detecting breast cancer. In Proceedings of the International Conference on Computer Science, Engineering and Applications, Chennai, India, 15-17 July 2011; pp. 37-45.

61. Happel, B.L.M.; Murre, J.M.J. The design and evolution of modular neural network architectures. Neural Netw. 1994, 7, 985-1004. [CrossRef]

62. Schapire, R.E. The strength of weak learnabilty. Mach. Learn. 1990, 5, 197-227. [CrossRef] 
63. Breiman, L. Bagging predictors. Mach. Learn. 1996, 24, 123-140. [CrossRef]

64. Bryll, R. Attribute bagging: Improving accuracy of classifier ensembles by using random feature subsets. Pattern Recognit. 2003, 36, 1291-1302. [CrossRef]

65. Ho, T.K. The Random Subspace Method for Constructing Decision Forests. IEEE Trans. Pattern Anal. Mach. Intell. 1998, 20, 832-844.

66. Bellman, R.E. Dynamic Programming; Courier Dover Publications: New York, NY, USA, 2003.

67. Freund, Y.; Schapire, R.E. Experiments with a New Boosting Algorithm; International Conference on Machine Learning: Bari, Italy, 1996; pp. 148-156.

68. Kohavi, R. A study of cross-validation and bootstrap for accuracy estimation and model selection. In Proceedings of the 14th International Joint Conference on Artificial Intelligence, Montreal, QC, Canada, 20-25 August 1995; pp. 1137-1143.

69. Kim, Y. Incheon Airport Welcomed 100 Million Passengers with 72 Automatic Immigration Control; Edaily: Seoul, Korea, 2017.

(C) 2019 by the authors. Licensee MDPI, Basel, Switzerland. This article is an open access article distributed under the terms and conditions of the Creative Commons Attribution (CC BY) license (http://creativecommons.org/licenses/by/4.0/). 